\title{
Current perspectives on atypical pneumonia in children
}

\author{
Jung Yeon Shim, MD \\ Department of Pediatrics, Kangbuk Samsung Hospital, Sungkyunkwan University of Medicine, Seoul, Korea
}

The major pathogens that cause atypical pneumonia are Mycoplasma pneumoniae, Chlamydophila pneumoniae, and Legionella pneumophila. Community-acquired pneumonia (CAP) caused by M. pneumoniae or C. pneumoniae is common in children and presents as a relatively mild and self-limiting disease. CAP due to L. pneumophila is very rare in children and progresses rapidly, with fatal outcomes if not treated early. $M$. pneumoniae, C. pneumoniae, and L. pneumophila have no cell walls; therefore, they do not respond to $\beta$-lactam antibiotics. Accordingly, macrolides, tetracyclines, and fluoroquinolones are the treatments of choice for atypical pneumonia. Macrolides are the first-line antibiotics used in children because of their low minimum inhibitory concentrations and high safety. The incidence of pneumonia caused by macrolide-resistant $M$. pneumoniae that harbors point mutations has been increasing since 2000, particularly in Korea, Japan, and China. The marked increase in macrolide-resistant $M$. pneumoniae pneumonia (MRMP) is partly attributed to the excessive use of macrolides. MRMP does not always lead to clinical nonresponsiveness to macrolides. Furthermore, severe complicated MRMP responds to corticosteroids without requiring a change in antibiotic. This implies that the hyper-inflammatory status of the host can induce clinically refractory pneumonia regardless of mutation. Empirical macrolide therapy in children with mild to moderate CAP, particularly during periods without M. pneumoniae epidemics, may not provide additional benefits over $\beta$-lactam monotherapy and can increase the risk of MRMP.

Key words: Pneumonia, Mycoplasma, Chlamydia, Legionella, Child

\section{Key message}

Macrolides are the first line treatment in atypical pneumonia caused by M. pneumoniae, C. pneumoniae, and L. pneumophila. Macrolide-resistant mycoplasma pneumonia (MRMP) is emerging worldwide, especially in East Asia. Immune modulators such as corticosteroids or second line antibiotics are treatment options for MRMP. Pediatricians should be careful with empirical therapy of macrolides in children with mild to moderate communityacquired pneumonia not to increase the risk of MRMP.

\section{Introduction}

Community-acquired pneumonia (CAP) is a common cause of morbidity and mortality in children. The term "atypical pneumonia" originates from its clinical features that differ from those of typical bacterial pneumonia caused by Streptococcus pneumoniae; it initially involves mild symptoms that progress to pneumonia with varying severity and extrapulmonary manifestations that do not respond to $\beta$-lactam antibiotics. ${ }^{1)}$ Atypical pathogens generally include all pathogens other than the typical bacteria. However, in narrow terms, Mycoplasma pneumoniae, Chlamydophila pneumoniae, and Legionella pneumophila are considered atypical pneumonia pathogens. These bacteria have several common findings; they cannot be isolated using routine microbiologic methods, are obligate or facultative intracellular parasites, and cause extrapulmonary symptoms. Moreover, since they do not have a peptidoglycan cell wall, they do not respond to $\beta$-lactam antibiotics. Instead, they show good responses to protein synthesis inhibitors, such as macrolides and tetracyclines (TCs), or DNA synthesis inhibitors such as fluoroquinolones (FQs). While pneumonia caused by $M$. pneumoniae and C. pneumoniae manifests as a mild, slowly progressing, and often self-limiting disease, that caused by L. pneumophila can present as abruptonset high fever and cough and rapidly progress to pleuritic chest pain, respiratory difficulty, and fatal outcomes if not treated early. ${ }^{2}$ Legionella infection can also cause Pontiac fever, a mild, self-limiting, flu-like disease that lasts $2-5$ days, which suggests that Legionella infection has various phenotypes like $M$. pneumoniae from asymptomatic infection to fatal pneumonia. There is an age-specific distribution of atypical pneumonia in which M. pneumoniae and C. pneumoniae pneumonia are more common in children aged $>3$ years, C. trachomatis pneumonia is more frequent in infants, and $L$. pneumophila pneumonia is very rare in children aged $<19$ years. ${ }^{3)}$ This might be related to the host's immune status and the bacterial colonization in the host as well as exposure to bacteria-contaminated settings. The incidence of M. pneumoniae pneumonia peaks in schoolaged children, thereafter decreasing in late adolescence and adulthood.

M. pneumoniae pneumonia accounts for $7 \%-20 \%$ of CAP

\footnotetext{
Corresponding author: Jung Yeon Shim, MD, PhD. Division of Pediatric Allergy \& Pulmonology, Department of Pediatrics, Kangbuk Samsung Hospital, Sungkyunkwan University School of Medicine, 29 Saemunan-ro, Jongro-gu, Seoul 03181, Korea

凶E-mail: jy7.shim@samsung.com, https://orcid.org/0000-0001-9367-2233

Received: 23 April, 2019, Revised: 19 February, 2020, Accepted: 28 February, 2020

This is an open-access article distributed under the terms of the Creative Commons Attribution Non-Commercial License (http://creativecommons.org/licenses/bync/4.0/) which permits unrestricted non-commercial use, distribution, and reproduction in any medium, provided the original work is properly cited.

Copyright (c) 2020 by The Korean Pediatric Society
} 
cases in children aged 3-15 years; an outbreak occurs every 3-4 years worldwide. C. pneumoniae pneumonia comprises 1\%-2\% of CAP cases in children. ${ }^{4}$ The diagnostic methods and criteria of C. pneumoniae pneumonia are not standardized; thus, its true incidence can be underestimated. ${ }^{5)} L$. pneumophila is a relatively rare etiology of CAP in children, accounting for less than $0.01 \%$ of pneumonia cases. $\left.{ }^{6}\right)$

Macrolides are the treatment of choice for atypical pneumonia because of their low minimum inhibitory concentration (MIC) and high safety profiles in children. However, the recent increasing incidence of macrolide-resistant M. pneumoniae pneumonia (MRMP) is becoming a worldwide problem, especially in Korea, Japan, and China. The point mutations of the $23 \mathrm{~S}$ rRNA genes (mostly at sites 2063, 2064, and 2617) inhibit the binding of macrolides to M. pneumoniae. ${ }^{7)}$ The increasing prevalence of MRMP may be attributed to the extensive use of macrolides because its incidence decreased with the reduced use of macrolides. ${ }^{8)}$ FQs or TCs are alternative antibiotics for patients with macrolide resistance; however, these drugs cause safety issues in children due to serious adverse reactions. The Korean Food and Drug Administration recommends the use of FQs and TCs in patients aged $>18$ years and $\geq 12$ years, respectively. ${ }^{7}$ Macrolide resistance among L. pneumophila and C. pneumoniae infections has been rarely reported; however, L. pneumophila with FQresistant mutations was reported recently. ${ }^{9)}$

Whether macrolides should be used as a first-line empirical therapy in children with CAP remains debated. The guidelines for the use of antibiotics in children with lower respiratory tract infections that are published by the Korea Centers for Disease Control and Prevention recommend using macrolides only when atypical pneumonia is suspected or confirmed. ${ }^{10)}$ How. ever, real-world macrolide prescriptions have not adhered to this guideline. Nonetheless, it may not be too late to begin macrolide treatment when atypical pathogens are suspected or confirmed in immunocompetent children with mild to moderate pneumonia. In contrast to L. pneumophila pneumonia, those due to $M$. pneumoniae and C. pneumoniae do not rapidly progress and are not life-threatening; rather, they usually progress slowly and present with self-limiting and benign courses. However, cases of fulminant MRMP and severe complications are reported occasionally. ${ }^{11)}$ L. pneumophila spreads from contaminated water, such as that from cooling towers, and is rarely observed in children. Legionellosis in Korean children aged $<19$ years reportedly occurs at a rate of $0.02 / 100,000$ individuals. ${ }^{12)}$ The extensive use of macrolides has increased the incidence of resistant strains, including M. pneumoniae and S. pneumoniae. MRMP disease severity does not significantly differ from that of macrolide-sensitive M. pneumoniae pneumonia (MSMP). ${ }^{13)}$ The clinical resistance to macrolides can be attributed to an excessive production of proinflammatory cytokines regardless of the mutation. Several studies have demonstrated the clinical effectiveness of corticosteroids in cases of MRMP that did not require a change of antibiotic. ${ }^{14,15)}$

This review assesses the epidemiology, pathogenesis, diagnosis, and treatment of atypical pneumonia caused by M. pneumoniae, C. pneumoniae, and L. pneumophila in children.

\section{Epidemiology}

The prevalence of atypical pneumonia varies by country and region, accounting for $10 \%-20 \%$ of all cases of CAP. In Korea, an infectious disease pathogen surveillance system was established in 2011 by the Korea Centers for Disease Control and Prevention to monitor M. pneumoniae and C. pneumoniae, while legionellosis monitoring has been in effect since 2001 . L. pneumophila pneumonia is extremely rare in children, with an incidence of $0.02 \%$ for every 100,000 individuals in Korean children aged $<19$ years (Fig. 1). ${ }^{12)}$ However, nosocomial Legionella outbreaks caused by contaminated cold mist humidifiers have been reported in the nurseries of term neonates. ${ }^{16}$ A total of nine term neonates were infected, three of whom died. In Korea, an L. pneumophila pneumonia outbreak occurred in the intensive care unit of a tertiary hospital in July 1986. A total of 23 patients were infected; of them, 4 died resulting in a mortality rate of $17 \%$. The contaminated cooling tower water of an air-conditioning system was identified as the source of the infection. The mode of L. pneumophila transmission is via the inhalation of aerosols or through the microaspiration of pathogens that are otherwise contained in portable hot water heaters, mist, evaporative condensers, cooling towers, humidifiers, and aerosol-generating apparatuses. There have been no reported cases of human-to-human infection.

The overall prevalence of mycoplasma pneumonia is higher in children than in adults. In Korea, M. pneumoniae pneumonia outbreaks occurred in 2011 and 2015, while a sporadic outbreak was observed in 2017-2018. ${ }^{12)}$ Genomic variation of the P1 protein and its firm adherence can lead to chronic carrier states or potential reactivation. However, whether this is due to reactivation or exposure to different genetic subtypes remains unclear. The incidence of C. pneumoniae pneumonia has been heterogeneous, ranging from $0.5 \%$ to greater than $5 \%$. A popu-

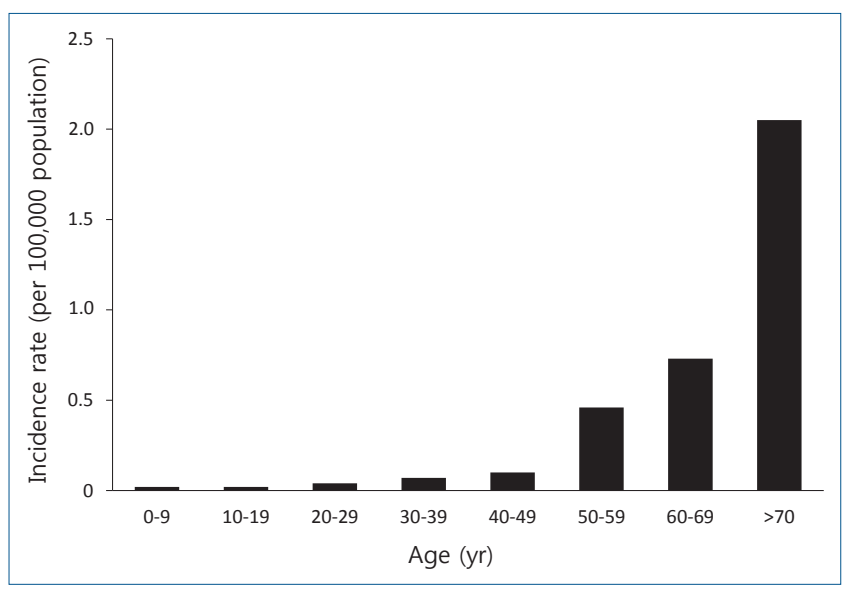

Fig. 1. Incidence of legionellosis by age, reported by the Korea Centers for Disease Control and Prevention in 2017. ${ }^{12)}$ 
lation-based surveillance of CAP cases requiring hospitalization was performed between 2010 and 2012 in the USA. ${ }^{17)}$ In this study, C. pneumoniae and M. pneumoniae were detected from nasopharyngeal or oropharyngeal samples using polymerase chain reaction (PCR). The incidence of C. pneumoniae and $M$. pneumoniae was $0.7 \%$ and 9.9\%, respectively, while that of $C$. pneumoniae and M. pneumoniae in hospitalized adult patients with CAP in a prospective multicenter study in Korea was 7.1\% and $6.3 \%$, respectively. ${ }^{18)}$

In contrast to M. pneumoniae pneumonia, there has been no clear yearly outbreak pattern for C. pneumoniae pneumonia (Fig. 2). ${ }^{12)}$ However, in Korea, the C. pneumoniae pneumonia incidence decreased by half, from 1,118 patients in 20152016 to 454 patients in 2017-2018, a similar trend to that of M. pneumoniae pneumonia. Around 20\% of children with C. pneumoniae pneumonia reportedly had M. pneumoniae coinfection, ${ }^{19)}$ although it is uncertain whether it is a true pathogen or colonization.

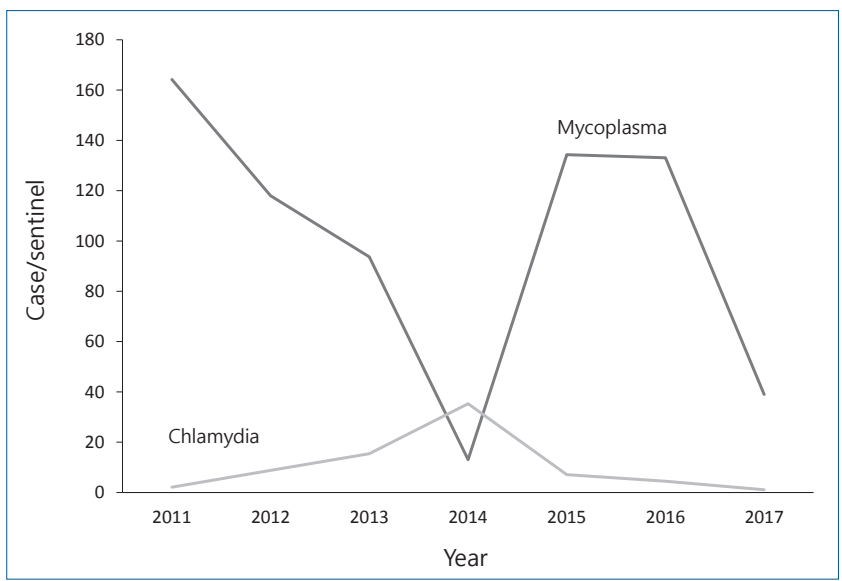

Fig. 2. Incidence of Mycoplasma pneumoniae and Chlamydophila pneumoniae infection in Korea from 2011 to 2017 reported by the Korea Centers for Disease Control and Prevention. ${ }^{12)}$
M. pneumoniae and C. pneumoniae can be transmitted quickly from human to human through close contact via large droplets. The incubation period is $2-4$ weeks; therefore, containment of mycoplasma pneumonia outbreaks takes a relatively long time.

\section{Pathophysiology}

The pathogenesis of M. pneumoniae infection is complicated, and the specific mechanism remains to be elucidated. $M$. pneumoniae makes close contact with the respiratory epithelium via firm attachment to the organelle or P1 proteins. The tight hostpathogen interactions within the respiratory epithelium lead to a direct injury by cytotoxic effects, local inflammation, host immune system stimulation, and sometimes extrapulmonary manifestations. ${ }^{20)}$

Inflammation by toll-like receptor (TLR)-mediated cytokine release and direct injury by reactive oxygen species (ROS) could be a major mechanism of mycoplasma infection (Fig. 3). Direct damage to the respiratory epithelium results from ROS such as $\mathrm{H}_{2} \mathrm{O}_{2}$ or superoxide produced by the organisms and the host's immune cells. Sodium transport in alveolar type II cells was inhibited by ROS-mediated injury during mycoplasma infection in a mouse model. ${ }^{21)}$ The host's inflammatory immune response can also lead to lung injury. Lipid-associated membrane protein of $M$. pneumoniae, such as macrophage-activating lipopeptide (MALP)-2, activates TLR-1, TLR-2, and TLR-6, which in turn activates nuclear factor- $\kappa \mathrm{B}(\mathrm{NF}-\kappa \mathrm{B})$ and induces proinflammatory cytokine release. ${ }^{22,23)}$ The community-acquired respiratory distress syndrome (CARDS) toxin released by $M$. pneumoniae enters alveolar macrophages and bronchial epithelium and activates NLR-family leucine-rich repeat protein (NLRP) 3 inflammasome, which in turn activates caspase- 1 and mediates the conversion of pro-interleukin (IL)- $1 \alpha$ into IL-1 $\beta$ and amplifies the inflammatory response; the mRNA expression

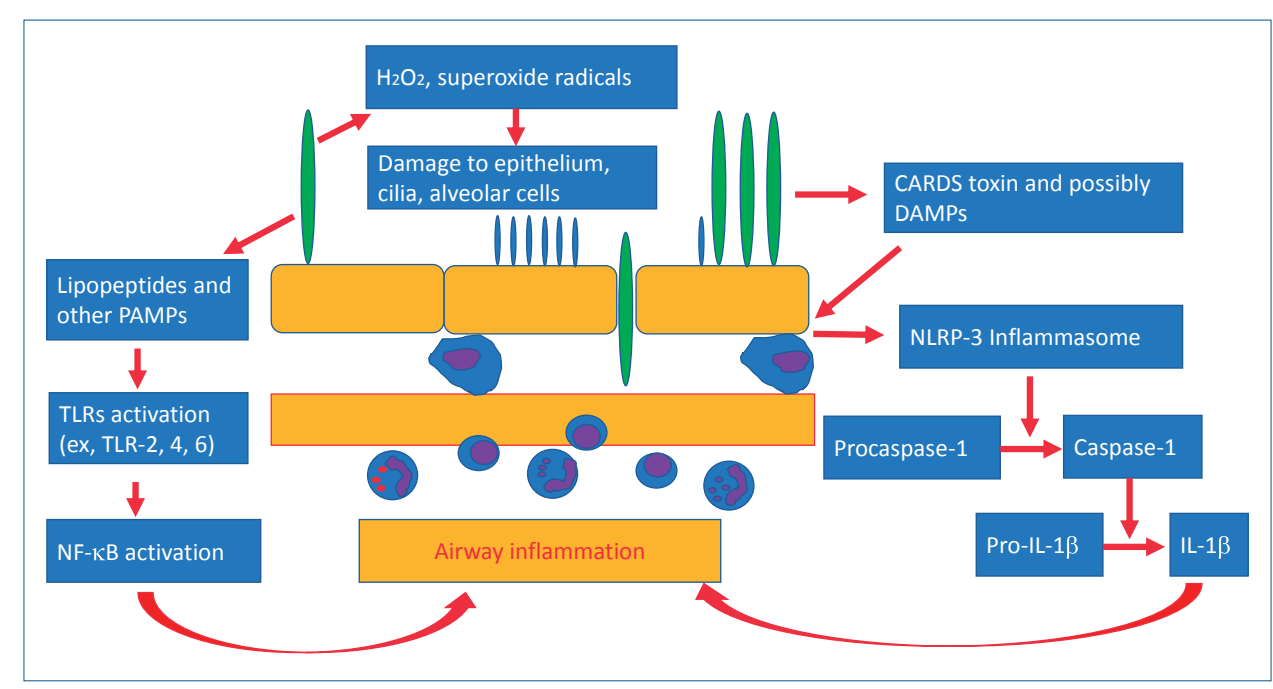

Fig. 3. Schematic diagram of pathogenesis induced by Mycoplasma pneumoniae. CARDS, communityacquired respiratory distress syndrome; DAMP, damage-associated molecular patterns; NF- B, nuclear factor kappa B; NLRP, NLR-family, leucine-rich repeat protein; PAMP, pathogen-associated molecular patterns; TLR, toll-like receptor; IL, interleukin. 
of tumor necrosis factor $\alpha$, IL 1 $\beta$, IL 6, IL 8, IL-18, inducible protein (IP)-10, and soluble IL 2 receptor levels increases in the lungs. ${ }^{24-26)}$ The excessive release of proinflammatory cytokines leads to host hyper-inflammation and tissue damage. Recombinant CARDS toxin can stimulate IL-4 and IL-13 production and increase airway reactivity and resistance in ovalbumin-sensitized mice model. ${ }^{27}$ ) In humans, mycoplasma infection also leads to acute asthma exacerbation in atopic children. It was recently suggested that fragments of pathogens, such as pathogenassociated molecular patterns, and substances from injured host cells, such as damage-associated molecular patterns, were involved in immune reactions of acute respiratory distress syndrome (ARDS) and pneumonia. ${ }^{28)}$ This can support the treatment effects of corticosteroids on a harmful, overreacting, cell-mediated immune response in ARDS or fulminant pneumonia caused by $M$. pneumoniae.$^{29,30)}$ The excessive host immune reactions can make the condition difficult to treat, prolong the fever, and delay the radiologic improvements in school-aged children and adolescents. Such conditions are rarely observed in infants.

M. pneumoniae can affect the red blood cells (RBCs), fibroblasts, and macrophages and alter the antigenicity of the $\mathrm{RBC}$ membranes or mimic the RBC membrane I antigen to induce autoantibody production and autoimmune hemolytic anemia. ${ }^{31)}$ M. pneumoniae membrane glycolipids also share common antigens with the brain, heart, and lung tissues, which causes the extrapulmonary manifestations. ${ }^{32)}$

Chlamydia has a unique developmental cycle in humans. The pathogen attaches to the host cells and undergoes endocytosis, differentiation, binary fission, and exocytosis or cytolysis. Chlamydia can persistently reside in host cells and cause prolonged subclinical infections. ${ }^{33)}$

Alveolar macrophages are the main target cells of Legionella. L. pneumophila replicates inside the macrophages until it kills them. Acute severe inflammation of the lungs can progress to necrosis. Host immune deficiency, chronic lung diseases, or corticosteroid therapy may create a high risk for L. pneumophila infections and poor outcomes. ${ }^{1)}$

\section{Diagnosis}

Diagnosis by culture is the most precise and specific method, but is very difficult, time-consuming, and limited to special laboratories. Serological tests are the most common diagnostic method but require time for antibody levels to increase. Antibodies can persist for months or even years after an infection, which may also cause false positive results. Molecular genetic diagnosis is fast and sensitive and most commonly involves PCR. The loop-mediated isothermal amplification method, which was much simpler and less expensive than PCR, was developed for gene amplification. ${ }^{34)}$ It can detect mutations in the 23S rRNA gene of M. pneumoniae with high sensitivity and specificity. The FilmArray Respiratory Panel, a highly multiplexed PCR approved by the US Food and Drug Administration, can detect
17 respiratory viruses and 3 bacteria (M. pneumoniae, $C$. pneumoniae, Bordetella pertussis) within 1-2 hours. ${ }^{35)}$ However, its detection rate depends on sample adequacy, and this method cannot differentiate normal flora from pathogens, particularly in nasopharyngeal samples. A rapid antigen kit using immunochromatography has been developed for a rapid diagnosis. ${ }^{36}$ ) How. ever, its exact sensitivity and specificity require confirmation.

The best method for diagnosing M. pneumoniae involves a combination of running a PCR using sputum or nasopharyngeal samples and serologic tests. ${ }^{37)}$ The diagnostic criteria require a 4-fold or greater increase in the antibody titer in the convalescent versus acute period. Immunofluorescence assays, enzyme-linked immunosorbent assays, and enzyme immunoassays can measure the mycoplasma-specific immunoglobulin $\mathrm{M}$ (IgM) and $\operatorname{IgG}$ levels. ${ }^{38)}$ The IgM antibody can be detected from approximately the first week of symptom onset, and its positivity can persist for $>1$ year after the infection, which causes false negative or positive results. Therefore, rising titer or positive conversion from negative to positive with 2 timepoint measurement is required for the early diagnosis. ${ }^{39)}$ In contrast to serology, PCR can detect organisms in the early stages of an infection. ${ }^{40)}$ How. ever, $21 \%$ of asymptomatic children tested positive for $M$. pneumoniae on PCR test during M. pneumoniae epidemics. ${ }^{41)}$ Therefore, a rising antibody titer in addition to running a PCR is necessary to discriminate carriage from infection.

The most common method for Legionella detection is the urinary antigen assay, a rapid and convenient test with high specificity (80\%) and sensitivity (99\%). This method can demonstrate positive results days after antibiotic treatment. This kit detects $L$. pneumophila serogroup 1, which accounts for $70 \%-80 \%$ of $L$. pneumophila pneumonia cases. ${ }^{42}$

No consensus has been reached on a standardized, validated, and accurate diagnostic method for C. pneumoniae. The serological test and PCR for $\mathrm{C}$. pneumoniae detection methodologies show low sensitivity and specificity. The first isolation of $C$. pneumoniae in Korea using PCR and an immunofluorescence test was reported in 2002.43) The antibody response to $C$. pneumoniae infection is very slow, making the diagnosis difficult; the diagnosis is usually retrospective. $\operatorname{IgM}$ antibody to $C$. pneumoniae tests positive 2 weeks after symptom onset, while IgG tests positive 3 weeks after symptom onset. ${ }^{44)}$ The US Centers for Disease Control and Prevention suggested modified strict serologic diagnostic criteria that use the microimmunofluorescence (MIF) test. Under these criteria, acute infections are defined by a 4-fold increase in the IgG titer or an $\operatorname{IgM}$ titer $\geq 16$; the use of a single elevated $\operatorname{IgG}$ titer is discouraged, and prior exposure is defined as an $\operatorname{IgG}$ titer $\geq 16 .{ }^{45)}$ However, the MIF test is likely to have low sensitivity because more than $50 \%$ of children with culture-proven C. pneumoniae infections have negative MIF antibody results. ${ }^{46}$ 


\section{Clinical manifestations}

Tracheobronchitis and pneumonia are the most common M. pneumoniae infections. The clinical symptoms are usually characterized by gradual-onset headache, malaise, fever, and sore throat, followed by cough. The cough usually worsens during the first week of the illness and resolves within 2 weeks, but it can last up to 4 weeks. Pneumonia is usually interstitial or bronchopneumonia; however, severe and complicated pneumonia, including parapneumonic effusion and necrotizing pneumonia, atelectasis, and bronchiolitis obliterans, have been reported. ${ }^{47}$

M. pneumoniae has been related to extrapulmonary diseases of many organ systems, including the skin, central nervous system (CNS), blood, heart, gastrointestinal tract, and joints. With improvements in PCR technology, the detection of $M$. pneumoniae DNA in nonrespiratory sites, particularly in the CNS, has become feasible. The most frequent extrapulmonary target organs are the CNS and skin.

The clinical manifestations of C. pneumoniae infections do not significantly differ from those of $M$. pneumoniae infections. A retrospective study of C. pneumoniae and M. pneumoniae infections in hospitalized Korean children with acute respiratory infections showed that rhinorrhea and pharyngeal injection were more common in C. pneumoniae infections than in $M$. pneumoniae infections. ${ }^{48}$ ) The cough is sometimes similar to that of a pertussis-like illness, and the asymptomatic carrier status can exceed 1 year. ${ }^{5)}$ The mean cough duration is 21 days; however, it can reach up to 60 days. The pneumonia is usually unilateral, and the bronchopneumonia mostly involves the lower lobes. Extrapulmonary manifestations are common, including gastrointestinal, neurologic, hepatitis, and skin.

In contrast to M. pneumoniae and C. pneumoniae pneumonia, which are self-limiting diseases, Legionella pneumonia is known to manifest as acute-onset high fever, productive cough, and chest pain and progress rapidly into various alveolar infiltrations that include unilateral or bilateral nodular, tumor-like infiltration, or cavitation. L. pneumophila pneumonia induces respiratory difficulty and altered consciousness. It also manifests as extrapulmonary symptoms, such as a headache, drowsiness, hyponatremia, elevated creatinine kinase, bradycardia, vomiting, diarrhea, abdominal pain, liver function abnormalities, and renal dysfunction. ${ }^{1)}$ Lung abscess, empyema, and rhabdomyolysis are common complications. The mortality rate can reach up to $30 \%$ if not treated early. ${ }^{1)}$

\section{Treatment}

The effective antibiotics and their daily doses and treatment periods are summarized in Table 1. M. pneumoniae responds to macrolides (erythromycin, roxithromycin, clarithromycin, and azithromycin), TCs, and FQs. The first-line antibiotics for $M$. pneumoniae infections in children are macrolides because they have a low MIC in MSMP. These drugs are protein synthesis inhibitors and have bacteriostatic activity. In contrast, FQs and TCs, the alternative antibiotics for MRMP, have relatively high MICs against MSMP and use limitations in children due to serious adverse effects. ${ }^{49)}$ Although macrolides are safe in children, they can cause side effects such as QT prolongation or arrhy. thmia. ${ }^{50)}$

It is recommended that clinicians consider the possibility of MRMP if patients with mycoplasma pneumonia show no response to macrolide treatment for 72 hours. TCs and FQs are alternative second-line antibiotics for MRMP; no refractory cases to TCs or FQs have been reported to date. TCs can induce permanent tooth discoloration by binding to the calcium to form an insoluble tetracycline-calcium orthophosphate complex. ${ }^{51)}$ Tetracycline derivatives, such as doxycycline or minocycline, also have this adverse effect. Although this has been demonstrated primarily in children with active mineralization, there have been a few reports in adults. ${ }^{52)} \mathrm{FQ}$ use is not allowed in children aged $<18$ years or without growth maturity because it can induce tendinopathy or tendon rupture with abrupt-onset sharp pain at rest or during movement. ${ }^{53)}$ However, there have been several controversial reports on its safety in children. ${ }^{54,55)}$

In Korea, TCs can be administered as alternative agents for children aged $\geq 12$ years, while FQs can be administered to children aged $>18$ years or with growth maturity.7) TCs or FQs should be used only when the benefits exceed the risks in children with MRMP.5) In Japan, minocycline (for children aged

Table 1. Treatment of atypical pneumonia

\begin{tabular}{|c|c|c|c|c|c|}
\hline Drug & Daily dose (mg/kg/day) & Divided/day & Route & Duration (day) & Maximum dose (mg/day) \\
\hline Erythromycin & $25-50$ & 4 & Oral & 14 & 4,000 \\
\hline Clarithromycin & $10-15$ & 2 & Oral & 10 & 1,000 \\
\hline Azithromycin & 10 & 1 & Oral & 3 & 500 \\
\hline Roxithromycin & $5-8$ & 2 & Oral & 10 & 300 \\
\hline Doxycycline & 4 & 2 & Oral & $7-14$ & 200 \\
\hline Minocycline & $2-4$ & 2 & Oral or intravenous & $7-14$ & 300 \\
\hline Levofloxacin & $\begin{array}{l}16-20 \text { in }<5 \text { years old } \\
810 \text { in } \geq 5 \text { years old }\end{array}$ & 2 & Oral & $7-14$ & 750 \\
\hline Tosufloxacin & 12 & 2 & Oral & $7-14$ & 360 \\
\hline
\end{tabular}

Levofloxacin and tosufloxacin should not be used routinely in children less than 18 years of age or without growth maturity. Doxycycline and minocycline should not be used routinely in children less than 12 years of age. 
$\geq 8$ years) and tosufloxacin (for children aged $<8$ years) have been approved to treat children with MRMP. ${ }^{57)}$ Doxycycline or minocycline administration is allowed for children aged $\geq 8$ years in Japan, Hong Kong, the US, and Europe. The use of FQs increased from $4.6 \%$ in 2010 to $22.6 \%$ in 2014, while macrolide use decreased from 62.8\% in 2010 to $50.6 \%$ in 2014 in Japan. ${ }^{58)}$ The prevalence of MRMP in Japan decreased from 81.6\% in 2012 to $43.6 \%$ in 2015, along with the decrease in macrolide use and the increase in FQ use. ${ }^{8)}$ As M. pneumoniae can easily become resistant to FQs through a single point mutation and FQs are broad-spectrum antibiotics, their use should be limited to children who have no other treatment options to prevent resistant strains. The prevalence of Mycoplasma genitalium with fluoroquinolone resistance-associated mutations is reportedly $10 \%-15 \%$ in Japan. ${ }^{59)}$

Other alternative treatments for MRMP in children include immunomodulatory agents such as systemic corticosteroids or intravenous immunoglobulin. They are most commonly used in cases of severe mycoplasma pneumonia or mycoplasma pneumonia with extrapulmonary manifestations, particularly with CNS involvement, Stevens-Johnson syndrome, and hemolytic anemia. ${ }^{11,14)}$ The fever usually subsided within 24 hours after the initiation of a systemic corticosteroid treatment and the radiologic abnormalities improved thereafter. ${ }^{60)}$ The indications and best timing of corticosteroid treatment as well as the optimal doses and durations in MRMP are yet to be determined. Doubleblind randomized placebo-controlled trials have shown that the early use of corticosteroids decreased the treatment failure rate without increasing complications and shortened the time for clinical stability in adult hospitalized patients with CAP of varying severities. ${ }^{61,62)}$ Studies have shown that proinflammatory cytokines such as IL- 6 and IL-8 produced in severe pneumonia were associated with high mortality rates in patients with $\mathrm{CAP}^{63)}$ A combination therapy of corticosteroids plus antibiotics reportedly decreased the cytokine levels and the bacterial burden better than antibiotics alone in an animal model of severe pneumonia. ${ }^{64)}$

The optimum dose of antibiotics and duration of treatment for C. pneumoniae infections remain uncertain. Macrolides, TCs, and quinolones show in vitro activity. Azithromycin and doxy. cycline have good activity against $C$. pneumoniae, while FQs are less active. Despite antibiotic therapy, coughing can persist for several weeks. ${ }^{65}$

Unlike for adults, L. pneumophila pneumonia is not considered in the differential diagnosis or empiric treatment for CAP in children. Azithromycin, clarithromycin, and the FQs are effective agents. In severe pneumonia or among immunocomromised children, initial parenteral therapy is recommended until the patients show a clinical response. The duration of therapy depends on the clinical severity and is usually 10-14 days. Corticosteroid treatment was reported to increase hospital mortality in L. pneumophila pneumonia. ${ }^{66}$ There have been no reports of macrolide-resistant strains of C. pneumoniae or $L$. pneumophila.
Debate persists about whether to use macrolides as a first-line treatment in children with CAP. A recent multicenter prospective observational study of children with CAP demonstrated that empirical therapy with a macrolide in combination with a $\beta$-lactam conferred no benefit over $\beta$-lactam monotherapy. ${ }^{67}$ However, 2 observational studies reported shorter lengths of stay for children with CAP who received combination therapies of macrolides plus $\beta$-lactams or ceftriaxone versus those who received $\beta$-lactam or ceftriaxone monotherapy. ${ }^{68,69)}$ Fisher's decision tree for high-risk M. pneumoniae in CAP is: (1) $>3$ years of age; and (2) fever lasting $>2$ days after the initiation of first-line antibiotics. The criteria recommend the administration of macrolides only to those with high-risk factors. ${ }^{70)}$ Most CAP guidelines suggest $\beta$-lactams as the first-line treatment of choice in children with mild to moderate CAP and the addition of macrolides only when atypical pneumonia is suggested or firstline antibiotics elicit no response. ${ }^{70-72)}$

\section{Conclusions}

The major pathogens that cause atypical pneumonia are $M$. pneumoniae, C. pneumoniae, and L. pneumophila. The treatments of choice for atypical pneumonia include macrolides, TCs, and FQs. Macrolides are the first-line antibiotics used in children. However, the extensive use of macrolides can lead to increased antibiotic resistance. It is recommended that macrolides be used only when atypical pneumonia is suspected or confirmed. A high risk of atypical pneumonia occurs in patients older than 3 years of age who do not respond to $\beta$-lactam antibiotics after more than 48-72 hours of treatment or in suspected cases of $L$. pneumophila pneumonia during an ongoing regional outbreak, with a travel history to an outbreak area, or in the presence of rapidly progressive pneumonia with extrapulmonary symptoms. A change of antibiotics to TCs and FQs or the addition of immunomodulatory agents to the regimen could be an alternative treatment for MRMP. TCs and FQs can be cautiously given to children less than 12 years of age and 18 years of age, respectively, if the benefits exceed the risks.

\section{Conflicts of interest}

No potential conflict of interest relevant to this article was reported.

\section{References}

1. Sharma L, Losier A, Tolbert T, Dela Cruz CS, Marion CR. Atypical pneumonia: updates on Legionella, Chlamydophila, and Mycoplasma pneumonia. Clin Chest Med 2017;38:45-58.

2. Yu Y, Fei A. Atypical pathogen infection in community-acquired pneumonia. Biosci Trends 2016;10:7-13.

3. Basarab M, Macrae MB, Curtis CM. Atypical pneumonia. Curr Opin Pulm Med 2014;20:247-51.

4. Don M, Canciani M, Korppi M. Community-acquired pneumonia in children: what's old? What's new? Acta Paediatr 2010;99:1602-8. 
5. Hammerschlag MR. Pneumonia due to Chlamydia pneumoniae in children: epidemiology, diagnosis, and treatment. Pediatr Pulmonol 2003; 36:384-90.

6. Phares CR, Wangroongsarb P, Chantra S, Paveenkitiporn W, Tondella ML, Benson RF, et al. Epidemiology of severe pneumonia caused by Legionella longbeachae, Mycoplasma pneumoniae, and Chlamydia pneumoniae: 1-year, population-based surveillance for severe pneumonia in Thailand. Clin Infect Dis 2007;45:e147-55.

7. Yang HJ, Song DJ, Shim JY. Mechanism of resistance acquisition and treatment of macrolide-resistant Mycoplasma pneumoniae pneumonia in children. Korean J Pediatr 2017;60:167-74.

8. Tanaka T, Oishi T, Miyata I, Wakabayashi S, Kono M, Ono S, et al. Macrolide-resistant Mycoplasma pneumoniae infection, Japan, 20082015. Emerg Infect Dis 2017;23:1703-6.

9. Shadoud L, Almahmoud I, Jarraud S, Etienne J, Larrat S, Schwebel C, et al. Hidden selection of bacterial resistance to fluoroquinolones in vivo: the case of Legionella pneumophila and humans. EBioMedicine 2015;2: 1179-85.

10. Korea Centers for Disease Control and Prevention (KCDC). 2017 Guidelines for the antibiotic use in children with lower respiratory tract infections [Internet]. Cheongju (Korea): KCDC; 2017 [cited 2019 Mar 5]. Available from: https://www.cdc.go.kr/board.es?mid=a2050702000 0\&bid $=0019 \&$ tag $=\&$ act $=$ view\&list_no $=138090$.

11. Izumikawa K. Clinical features of severe or fatal Mycoplasma pneumoniae pneumonia. Front Microbiol 2016;7:800.

12. Korea Centers for Disease Control and Prevention (KCDC). Infectious disease portal. Infectious disease surveillance yearbook 2017 [Internet]. Cheongju (Korea): KCDC; 2017 [cited 2019 Mar 5]. Available from: http://www.cdc.go.kr/npt/biz/npp/portal/nppPblctDtaMain.do.

13. Matsubara K, Morozumi M, Okada T, Matsushima T, Komiyama O, Shoji M, et al. A comparative clinical study of macrolide-sensitive and macrolide-resistant Mycoplasma pneumoniae infections in pediatric patients. J Infect Chemother 2009;15:380-3.

14. Shen Y, Zhang J, Hu Y, Shen K. Combination therapy with immunemodulators and moxifloxacin on fulminant macrolide-resistant Mycoplasma pneumoniae infection: a case report. Pediatr Pulmonol 2013;48:51922.

15. Luo Z, Luo J, Liu E, Xu X, Liu Y, Zeng F, et al. Effects of prednisolone on refractory mycoplasma pneumoniae pneumonia in children. Pediatr Pulmonol 2014;49:377-80.

16. Yiallouros PK, Papadouri T, Karaoli C, Papamichael E, Zeniou M, Pieridou-Bagatzouni D, et al. First outbreak of nosocomial Legionella infection in term neonates caused by a cold mist ultrasonic humidifier. Clin Infect Dis 2013;57:48-56.

17. Jain S, Self WH, Wunderink RG; CDC EPIC Study Team. Communityacquired pneumonia requiring hospitalization. N Engl J Med 2015;373: 2382.

18. Sohn JW, Park SC, Choi YH, Woo HJ, Cho YK, Lee JS, et al. Atypical pathogens as etiologic agents in hospitalized patients with communityacquired pneumonia in Korea: a prospective multi-center study. J Korean Med Sci 2006;21:602-7.

19. Hammerschlag MR. Chlamydia trachomatis and Chlamydia pneumoniae infections in children and adolescents. Pediatr Rev 2004;25:43-51.

20. Meyer Sauteur PM, Unger WW, Nadal D, Berger C, Vink C, van Rossum AM. Infection with and carriage of Mycoplasma pneumoniae in children. Front Microbiol 2016;7:329.

21. Hickman-Davis JM, McNicholas-Bevensee C, Davis IC, Ma HP, Davis GC, Bosworth CA, et al. Reactive species mediate inhibition of alveolar type II sodium transport during mycoplasma infection. Am J Respir Crit Care Med 2006;173:334-44.

22. Saraya T, Nakata K, Nakagaki K, Motoi N, lihara K, Fujioka Y, et al. Identification of a mechanism for lung inflammation caused by Mycoplasma pneumoniae using a novel mouse model. Results Immunol 2011; 1:76-87.

23. Shimizu T, Kida Y, Kuwano K. A dipalmitoylated lipoprotein from Mycoplasma pneumoniae activates NF-kappa B through TLR1, TLR2, and TLR6. J Immunol 2005;175:4641-6.
24. Yang J, Hooper WC, Phillips DJ, Talkington DF. Cytokines in Mycoplasma pneumoniae infections. Cytokine Growth Factor Rev 2004;15:157-68.

25. Becker A, Kannan TR, Taylor AB, Pakhomova ON, Zhang Y, Somarajan SR, et al. Structure of CARDS toxin, a unique ADP-ribosylating and vacuolating cytotoxin from Mycoplasma pneumoniae. Proc Natl Acad Sci US A 2015;112:5165-70.

26. Baroja-Mazo A, Martín-Sánchez F, Gomez AI, Martínez CM, AmoresIniesta J, Compan V, et al. The NLRP3 inflammasome is released as a particulate danger signal that amplifies the inflammatory response. Nat Immunol 2014;15:738-48.

27. Medina JL, Coalson JJ, Brooks EG, Le Saux CJ, Winter VT, Chaparro A, et al. Mycoplasma pneumoniae CARDS toxin exacerbates ovalbumininduced asthma-like inflammation in BALB/c mice. PLoS One 2014;9: e102613.

28. Lee KY. Pneumonia, acute respiratory distress syndrome, and early immune-modulator therapy. Int J Mol Sci 2017;18:388.

29. Saraya T, Kurai D, Nakagaki K, Sasaki Y, Niwa S, Tsukagoshi H, et al. Novel aspects on the pathogenesis of Mycoplasma pneumoniae pneumonia and therapeutic implications. Front Microbiol 2014;5:410.

30. Yang EA, Kang HM, Rhim JW, Kang JH, Lee KY. Early corticosteroid therapy for Mycoplasma pneumoniae pneumonia irrespective of used antibiotics in children. J Clin Med 2019;8:726.

31. Chen CJ, Juan CJ, Hsu ML, Lai YS, Lin SP, Cheng SN. Mycoplasma pneumoniae infection presenting as neutropenia, thrombocytopenia, and acute hepatitis in a child. J Microbiol Immunol Infect 2004;37:128-30.

32. Fernald GW. Immunologic mechanisms suggested in the association of $M$. pneumoniae infection and extrapulmonary disease: a review. Yale J Biol Med 1983;56:475-9.

33. Bastidas RJ, Elwell CA, Engel JN, Valdivia RH. Chlamydial intracellular survival strategies. Cold Spring Harb Perspect Med 2013;3:a010256.

34. Gotoh K, Nishimura N, Ohshima Y, Arakawa Y, Hosono H, Yamamoto Y, et al. Detection of Mycoplasma pneumoniae by loop-mediated isothermal amplification (LAMP) assay and serology in pediatric communityacquired pneumonia. J Infect Chemother 2012;18:662-7.

35. Chen H, Weng H, Lin M, He P, Li Y, Xie Q, et al. The clinical significance of FilmArray respiratory panel in diagnosing community-acquired pneumonia. Biomed Res Int 2017;2017:7320859.

36. Ou L, Lv Q, Wu C, Hao H, Zheng Y, Jiang Y. Development of a lateral flow immunochromatographic assay for rapid detection of Mycoplasma pneumoniae-specific IgM in human serum specimens. J Microbiol Methods 2016;124:35-40.

37. Medjo B, Atanaskovic-Markovic M, Radic S, Nikolic D, Lukac M, Djukic S. Mycoplasma pneumoniae as a causative agent of community-acquired pneumonia in children: clinical features and laboratory diagnosis. Ital J Pediatr 2014;40:104.

38. Yoo SJ, Oh HJ, Shin BM. Evaluation of four commercial IgG- and IgMspecific enzyme immunoassays for detecting Mycoplasma pneumoniae antibody: comparison with particle agglutination assay. J Korean Med Sci 2007;22:795-801.

39. Lee SC, Youn YS, Rhim JW, Kang JH, Lee KY. Early serologic diagnosis of Mycoplasma pneumoniae pneumonia: an observational study on changes in titers of specific-IgM antibodies and cold agglutinins. Medicine (Baltimore) 2016;95:e3605.

40. Chang HY, Chang LY, Shao PL, Lee PI, Chen JM, Lee CY, et al. Comparison of real-time polymerase chain reaction and serological tests for the confirmation of Mycoplasma pneumoniae infection in children with clinical diagnosis of atypical pneumonia. J Microbiol Immunol Infect 2014;47:137-44.

41. Spuesens EB, Fraaij PL, Visser EG, Hoogenboezem T, Hop WC, van Adrichem LN, et al. Carriage of Mycoplasma pneumoniae in the upper respiratory tract of symptomatic and asymptomatic children: an observational study. PLoS Med 2013;10:e1001444.

42. Beraud L, Gervasoni K, Freydiere AM, Descours G, Ranc AG, Vandenesch F, et al. Comparison of Sofia Legionella FIA and BinaxNOW ${ }^{\circledR}$ Legionella urinary antigen card in two national reference centers. Eur J Clin Microbiol Infect Dis 2015;34:1803-7.

43. Lee SJ, Jung HH, Kim SK, Choi DH, Han SS, Nam EC, et al. The first 
isolation of Chlamydia pneumoniae from a Korean patient. Tuberc Respir Dis 2002;53:569-76.

44. Miyashita N, Kawai Y, Tanaka T, Akaike H, Teranishi H, Wakabayashi T, et al. Antibody responses of Chlamydophila pneumoniae pneumonia: why is the diagnosis of C. pneumoniae pneumonia difficult? J Infect Chemother 2015;21:497-501.

45. Dowell SF, Peeling RW, Boman J, Carlone GM, Fields BS, Guarner J, et al. Standardizing Chlamydia pneumoniae assays: recommendations from the Centers for Disease Control and Prevention (USA) and the Laboratory Centre for Disease Control (Canada). Clin Infect Dis 2001;33:492-503.

46. Pierce VM, Elkan M, Leet M, McGowan KL, Hodinka RL. Comparison of the Idaho Technology FilmArray system to real-time PCR for detection of respiratory pathogens in children. J Clin Microbiol 2012;50:364-71.

47. LiYN, Liu L, Qiao HM, Cheng H, Cheng HJ. Post-infectious bronchiolitis obliterans in children: a review of 42 cases. BMC Pediatr 2014;14:238.

48. Sung JJ, Kim EJ, Sun YH, Jeon IS, Tchah H, Ryoo E, et al. Clinical presentations of Chlamydia pneumoniae in children hospitalized for acute respiratory infections: a comparison to Mycoplasma pneumonia. Allergy Asthma Respir Dis 2015;3:346-51.

49. Smith CB, Friedewald WT, Chanock RM. Shedding of Mycoplasma pneumoniae after tetracycline and erythromycin therapy. N Engl J Med 1967;276:1172-5.

50. Germanakis I, Galanakis E, Parthenakis F, Vardas PE, Kalmanti M. Clarithromycin treatment and QT prolongation in childhood. Acta Paediatr 2006;95:1694-6.

51. Sánchez AR, Rogers RS 3rd, Sheridan PJ. Tetracycline and other tetracy. cline-derivative staining of the teeth and oral cavity. Int J Dermatol 2004; 43:709-15.

52. McKenna BE, Lamey PJ, Kennedy JG, Bateson J. Minocycline-induced staining of the adult permanent dentition: a review of the literature and report of a case. Dent Update 1999;26:160-2.

53. Szarfman A, Chen M, Blum MD. More on fluoroquinolone antibiotics and tendon rupture. N Engl J Med 1995;332:193.

54. Rosanova MT, Lede R, Capurro H, Petrungaro V, Copertari P. Assessing fluoroquinolones as risk factor for musculoskeletal disorders in children: a systematic review and meta-analysis. Arch Argent Pediatr 2010;108:52431.

55. Forsythe CT, Ernst ME. Do fluoroquinolones commonly cause arthropathy in children? CJEM 2007;9:459-62.

56. Jackson MA, Schutze GE; Committee on Infectious Diseases. The use of systemic and topical fluoroquinolones. Pediatrics 2016;138:e20162706.

57. Yamazaki T, Kenri T. Epidemiology of Mycoplasma pneumoniae infections in Japan and therapeutic strategies for macrolide-resistant $M$. pneumoniae. Front Microbiol 2016;7:693.

58. Okubo Y, Michihata N, Morisaki N, Uda K, Miyairi I, Ogawa Y, et al. Recent trends in practice patterns and impact of corticosteroid use on pediatric Mycoplasma pneumoniae-related respiratory infections. Respir Investig 2018;56:158-65

59. Deguchi T, Ito S, Yasuda M, Sato Y, Uchida C, Sawamura M, et al. Surveillance of the prevalence of macrolide and/or fluoroquinolone resistance-associated mutations in Mycoplasma genitalium in Japan. J Infect Chemother 2018;24:861-7.
60. Tamura A, Matsubara K, Tanaka T, Nigami H, Yura K, Fukaya T. Methylprednisolone pulse therapy for refractory Mycoplasma pneumoniae pneumonia in children. J Infect 2008;57:223-8.

61. Blum CA, Nigro N, Briel M, Schuetz P, Ullmer E, Suter-Widmer I, et al. Adjunct prednisone therapy for patients with community-acquired pneumonia: a multicentre, double-blind, randomised, placebo-controlled trial. Lancet 2015;385:1511-8.

62. Torres A, Sibila O, Ferrer M, Polverino E, Menendez R, Mensa J, et al. Effect of corticosteroids on treatment failure among hospitalized patients with severe community-acquired pneumonia and high inflammatory response: a randomized clinical trial. JAMA 2015;313:677-86.

63. Martínez R, Menéndez R, Reyes S, Polverino E, Cillóniz C, Martínez A, et al. Factors associated with inflammatory cytokine patterns in communityacquired pneumonia. Eur Respir J 2011;37:393-9.

64. Sibila O, Luna CM, Agustí C, Baquero S, Gando S, Patrón JR, et al. Effects of glucocorticoids in ventilated piglets with severe pneumonia. Eur Respir J 2008;32:1037-46.

65. Hammerschlag MR, Chirgwin K, Roblin PM, Gelling M, Dumornay W, Mandel L, et al. Persistent infection with Chlamydia pneumoniae following acute respiratory illness. Clin Infect Dis 1992;14:178-82.

66. Chidiac $\mathrm{C}$, Che $\mathrm{D}$, Pires-Cronenberger $\mathrm{S}$, Jarraud $\mathrm{S}$, Campèse $\mathrm{C}$, Bissery $\mathrm{A}$, et al. Factors associated with hospital mortality in community-acquired legionellosis in France. Eur Respir J 2012;39:963-70.

67. Williams DJ, Edwards KM, Self WH, Zhu Y, Arnold SR, McCullers JA, et al. Effectiveness of $\beta$-lactam monotherapy vs macrolide combination therapy for children hospitalized with pneumonia. JAMA Pediatr 2017; 171:1184-91.

68. Ambroggio L, Taylor JA, Tabb LP, Newschaffer CJ, Evans AA, Shah SS. Comparative effectiveness of empiric $\beta$-lactam monotherapy and $\beta$-lactam-macrolide combination therapy in children hospitalized with community-acquired pneumonia. J Pediatr 2012;161:1097-103.

69. Leyenaar JK, Shieh MS, Lagu T, Pekow PS, Lindenauer PK. Comparative effectiveness of ceftriaxone in combination with a macrolide compared with ceftriaxone alone for pediatric patients hospitalized with communityacquired pneumonia. Pediatr Infect Dis J 2014;33:387-92.

70. Bandell RA, Dekkers T, Semmekrot BA, de Wildt SN, Fleuren HW, Warlévan Herwaarden MF, et al. Macrolide prescription in Dutch children: compliance with guidelines. Eur J Clin Microbiol Infect Dis 2019;38:67581.

71. Mathur S, Fuchs A, Bielicki J, Van Den Anker J, Sharland M. Antibiotic use for community-acquired pneumonia in neonates and children: WHO evidence review. Paediatr Int Child Health 2018;38(sup1):S66-75.

72. Uehara S, Sunakawa K, Eguchi H, Ouchi K, Okada K, Kurosaki T, et al. Japanese guidelines for the management of respiratory infectious diseases in children 2007 with focus on pneumonia. Pediatr Int 2011;53:264-76.

How to cite this article: Shim JY. Current perspectives on atypical pneumonia in children. Clin Exp Pediatr 2020;63:46976. https://doi.org/10.3345/cep.2019.00360 